\title{
Can industry regulators learn collusion structures from information-efficient asset markets?*
}

\author{
Alexander Zimper ${ }^{\dagger} \quad$ Shakill Hassan ${ }^{\ddagger}$
}

December 22, 2011

\begin{abstract}
This note combines a dynamic industrial organization model, in which an industry is subject to exogenous processes of market-size and collusion structure, with a consumption-based asset pricing model for the shares in the industry's firms. Three main findings emerge for our model under the assumption of informationefficient asset markets. Firstly, the volatility of a firm's share price is exclusively driven by the volatility of the industry's market-size. Secondly, the volatility of a firm's price-dividend ratio is exclusively driven by the volatility of the industry's collusion structure whereby high (resp. low) ratios indicate less (resp. more) collusion. Thirdly, for non-volatile collusion structures the price-dividend ratio is constant across different collusion structures.
\end{abstract}

Keywords: Cournot interaction; collusion; price-dividend ratio; consumptionbased asset pricing

JEL Classification Numbers: G12; L10; L50

*We thank Maxim Engers, Roman Inderst and Alexander Ludwig for helpful comments and suggestions.

${ }^{\dagger}$ Corresponding author. Department of Economics, University of Pretoria, Private Bag X20, Hatfield 0028, South Africa. E-mail: alexander.zimper@up.ac.za

${ }^{\ddagger}$ South African Reserve Bank and School of Economics, University of Cape Town, Private Bag, Rondebosch, South Africa; Email: shakill.hassan@uct.ac.za. 


\section{Introduction}

Since a regulator has typically no direct information about an industry's collusion structure, it is standard for regulatory institutions to look at product-prices and profits as possible indicators for collusion. ${ }^{1}$ In the relevant case in which the regulator cannot directly observe the industry's demand fluctuations, however, the identification problem arises whether changes in product-prices and profits are rather caused by changes in the collusion structure or by changes in the industry's demand-side.

In this technical note we take the information-efficient financial markets hypothesis $(=\mathrm{EMH})$ seriously and ask the following question: Can a regulator learn the industry's collusion structure by simply observing publicly available financial market data such as the firms' share prices and dividend-payments? To address this question, we develop a closed-form model of asset-pricing for the shares in a representative firm that operates in an economic environment in which the industry's market size and its collusion structure are subject to exogenous stochastic processes. More specifically, we combine a gametheoretic model of an infinite sequence of Cournot competition games with a Lucas (1978) type consumption-based infinite horizon asset pricing model. The industry's demand-side is modelled through an exogenous stochastic process according to which the industry's market size growth rate is normally distributed. In the asset pricing model we stick to the standard assumptions (cf., e.g., Mehra and Prescott 1985) according to which the representative investor has a CRRA utility function and consumption growth is normally distributed. Compared to the existing asset-pricing literature, our model adds the new twist that a firm's profit, i.e., dividend payments, is determined by the industry's market size as well as its collusion structure.

Under convenient (independence) assumptions on the joint distribution of the consumption growth-, the market-size-, and the collusion structure processes, three main findings emerge for our model:

1. The volatility of a firm's share price is exclusively driven by the volatility of the industry's market-size.

\footnotetext{
${ }^{1}$ Bolotova, Connor and Miller (2008) offer a rare examination of the time series properties of prices before, during, and after cartelization (of the citric acid industry). The cartel initially controlled prices as well as quantities but competition from Chinese imports effectively removed domestic incumbents' control over market prices. Data in Bolotova, Connor and Miller (2008) (page 1295, Table 1) show that the mean price level prior to cartel formation was 65.35 . This increased to 75.96 during the cartel's operation (an increase of 16 percent).
} 
2. The volatility of a firm's price-dividend ratio is exclusively driven by the volatility of the industry's collusion structure whereby less (more) collusion in any given period implies a higher (lower) price-dividend ratio for this period.

3. For time-invariant collusion structures the price-dividend ratio is identical across all different collusion structures.

According to these findings neither share prices nor price-dividend ratios for nonvolatile collusion structures are an indicator for an industry's collusion structure. However, whenever an industry's collusion structure is volatile, high (resp. low) pricedividend ratios are indicators for low (resp. high) collusion in this industry. While the above findings are obtained within a highly stylized theoretical framework, we hope that they might guide future empirical investigations that explicitly look at the relationship between volatile price-dividend ratios at firm level, on the one hand, and different degrees of competition within the industry, on the other hand.

\section{Cournot interaction with exogenous collusion struc- ture}

We construct an infinite sequence of Cournot competition games that are subject to different market-sizes as well as different structures of industrial collusion over time. Both processes - the market-size process and the collusion process - are exogenous to the model in the sense that at each time period nature determines market-size and the collusion structure. That is, in contrast to typical models on collusion in the industrial organization literature we do not explicitly model collusion as a strategic choice of firms. Rather we think of the exogenous collusion process as either caused by changes in exogenous political variables (e.g., cartel legislature or effectiveness of the competition commission) or by changes in the firms' management policies (e.g., fluctuations of CEOs with different appetites for risk or for empire-building).

Formally, we consider a finite set of infinitely lived firms $\{A, B, \ldots\}$ with $\#\{A, B, \ldots\}=$ $n$. Any partition of $N$ is a collusion structure whereby $\mathcal{S}$ denotes the set of all possible collusion structures, (i.e., $\mathcal{S}$ collects all partitions of $N$ ), with its cardinality given as the so-called Bell number ${ }^{2} . I_{t} \in \mathcal{S}$ denotes the collusion structure of the industry at period $t$ with cardinality $\# I_{t}$. A member $i \in I_{t}$, i.e., some non-empty subset of firms in $\{A, B, \ldots\}$, is called an alliance. Finally, define by $\# i^{A}: \mathcal{S} \rightarrow\{1,2, \ldots, n\}$ the function

\footnotetext{
${ }^{2}$ Note that the first ten Bell numbers are: 1, 2, $5,15,52,203,877,4140,21147,115975$, i.e., there are 115975 different ways to partition a set that contains ten members.
} 
that counts for any given alliance $I_{t} \in \mathcal{S}$ the total number of firms belonging to the same alliance as firm $A$.

Example. Suppose that there are three firms in the industry, i.e., $\{A, B, C\}$. For $n=3$ the cardinality (=Bell number) of $\mathcal{S}$ is 5 whereby

\begin{tabular}{|l|l|l|l|}
\hline & $I_{t}$ & $\# I_{t}$ & $\# i^{A}$ \\
\hline 1 & $\{\{A\},\{B\},\{C\}\}$ & 3 & 1 \\
\hline 2 & $\{\{A, B\},\{C\}\}$ & 2 & 2 \\
\hline 3 & $\{\{A, C\},\{B\}\}$ & 2 & 2 \\
\hline 4 & $\{\{A\},\{B, C\}\}$ & 2 & 1 \\
\hline 5 & $\{\{A, B, C\}\}$ & 1 & 3 \\
\hline
\end{tabular}

Definition. Consider the following period $t$ Cournot-competition game $G_{t}=\left\langle S_{i}, U_{i}\right\rangle_{i \in I_{t}}$ such that, for all $i \in I_{t}, S_{i}=R_{+}$and $U_{i}: \times_{i=1}^{\# I_{t}} S_{i} \rightarrow R_{+}$is defined as

$$
U_{i}\left(s_{i}, s_{-i}\right)=\max \left\{0,\left(X_{t}-\sum_{j=1}^{\# I_{t}} s_{j}\right)\right\} \cdot s_{i}
$$

where the market-size in period $t, X_{t} \geq 0$, stands for the linear inverse demand function's intercept.

According to the above definition, the alliances of collusion structure $I_{t}$ act as players who maximize their utility (=profit) within a simple one-period model of linear Cournotcompetition. In this game's unique Nash equilibrium $s^{*} \in S$ the profit of any alliance $i \in I_{t}$ is given by

$$
U_{i}\left(s^{*}\right)=\left(\frac{X_{t}}{\# I_{t}+1}\right)^{2} .
$$

Observation 1. Under the assumption that the profit of any alliance is equally divided among its colluding firms, the profit of firm $A$ is given as the adapted stochastic process

$$
Y_{t}=\frac{X_{t}^{2}}{K_{t}}, t \geq 1
$$

such that the random variable

$$
K_{t}=\# i^{A}\left(I_{t}\right) \cdot\left(\# I_{t}+1\right)^{2}
$$

is completely determined by the industry's collusion structure. 
Remark. The industrial organization literature typically uses different models of strategic interaction in order to represent different degrees of competitive intensity. Namely, Bertrand interaction is used to represent more intense competition (low prices) whereas Cournot interaction represents softer competition (higher prices), (cf., e.g., Tirole 1988; Bester and Petrakis 1993; Bonanno and Haworth 1998). While such interpretation is useful and natural when contrasting different industries, it is of rather limited use when analyzing dynamics within a given industry. In contrast, we define the intensity of competition in terms of the number of alliances within that industry. In the extreme case where the number of alliances equals the total number of firms in the industry, i.e., $\# I_{t}=n$, we have the most intensively competitive outcome, associated with low profits. In the other extreme, i.e., $\# I_{t}=1$, the entire industry forms one big alliance (i.e., a monopoly or cartel) corresponding to the least competitive case. Time variation in the number of such alliances, which we model as an exogenous stochastic process, determines the intensity of competition in the industry. In this sense, our definition of competitive intensity permits us to model variations in the intensity of competition within an industry over time.

\section{The asset price equilibrium}

By Observation 1, the period $t$ profit (2) of firm $A$ is completely determined by the period $t$ market-size $X_{t}$ and by the industry's collusion structure $I_{t}$ expressed through $K_{t}$. In this section we derive a closed-form solution for period $t$ asset prices of shares in firm $A$. To this end we consider a consumption-based asset pricing model with an infinitely lived representative investor who has in every time period perfect insider information about the Cournot industry; that is, the investor knows the market-size as well as the industry's collusion structure.

More specifically, we assume that the representative period $t$ investor has standard additively separable CRRA preferences over future consumption streams such that for all $s \geq t$

$$
U\left(C_{s}\right)=\ln C_{s},
$$

where $C_{s}$ denotes the random variable for the consumption level in period $s \geq t$. The representative period $t$ investor chooses asset holdings $x_{s}$ in the firm's shares for periods $s \geq t$ as the solution to the maximization problem

$$
\max _{\left(x_{t}, x_{t+1, \ldots}\right),}\left(U\left(C_{t}\right)+\sum_{s=t+1}^{\infty} E_{t}\left[\beta^{s-t} \cdot U\left(C_{s}\right)\right]\right)
$$


where $\beta=e^{-\delta}<1$ denotes the investor's time-discount factor and $E_{t}\left[U\left(C_{s}\right)\right]$ denotes the investor's expected utility of period $s$ consumption with respect to a conditional probability measure $\pi_{t}$ taking into account the investor's information at period $t$. The budget condition is given by

$$
C_{s}=W_{s}+Y_{s} \cdot x_{s}+P_{s} \cdot\left(x_{s}-x_{s+1}\right) \text { for all } s
$$

where $W_{s}$ denotes (random) period $s$ income not resulting from the asset's dividend payments and $P_{s}$ denotes the ex-profit asset price in period $s$. We further assume that the period $s$ dividend payment $Y_{s}$ coincides with the period $s$ profit of firm $A$ as given by $(2)$.

The corresponding Euler equations - stating necessary first order conditions for equilibrium prices - are

$$
P_{t}^{*}=E_{t}\left[M_{t, t+1} \cdot\left(Y_{t+1}+P_{t+1}^{*}\right)\right] \text { for } t=0,1, \ldots
$$

whereby

$$
M_{t, s}=\beta^{s-t} \cdot\left(\frac{C_{s}}{C_{t}}\right)^{-1}
$$

denotes the stochastic discount factor for $s>t$. It is standard to show that the above Euler equations - combined with a transversality condition requiring that $\lim _{s \rightarrow \infty} E_{t}\left[M_{t, s} \cdot P_{s}^{*}\right]=$ 0 - give rise to the following period $t$ equilibrium prices for the asset

$$
P_{t}^{*}=\sum_{s=t+1}^{\infty} E_{t}\left[M_{t, s} \cdot Y_{s}\right] .
$$

To derive a convenient closed-form solution to (7), we impose further structure on the three exogenous stochastic processes - $\left(C_{t}\right)_{t \geq 1},\left(X_{t}\right)_{t \geq 1}$, and $\left(K_{t}\right)_{t \geq 1}$ - that drive our model. To this end define the state space

$$
\Omega=\times_{s=0}^{\infty}\left(\mathbb{R}_{+} \times \mathbb{R}_{+} \times \mathbb{R}_{+}\right)
$$

and let $C_{t}, X_{t}$, and $K_{t}$ denote the corresponding coordinate variables. Denote by $\mathcal{F}_{t}$, $t \geq 1$, the Borel $\sigma$-algebra on

$$
\times_{s=0}^{t}\left(\mathbb{R}_{+} \times \mathbb{R}_{+} \times \mathbb{R}_{+}\right)
$$

and consider henceforth the probability space $(\pi, \mathcal{F}, \Omega)$ where $\mathcal{F}$ denotes the standard product algebra generated by all $\mathcal{F}_{t}$. Further suppose that the probability measure $\pi$ satisfies the following assumptions about the joint distributions of adapted stochastic processes. 


\section{Distributional assumptions.}

(A1) The continuously compounded growth rates of consumption, i.e., $g_{C, t}$ such that

$$
e^{g_{C, t}}=\frac{C_{t}}{C_{t-1}}
$$

are i.i.d. over time such that, for all $t$,

$$
g_{C, t}=g_{C} \text { with } g_{C} \sim N\left(\mu_{g_{C}}, \sigma_{g_{C}}^{2}\right)
$$

(A2) The continuously compounded growth rates of the market-size, i.e., $g_{X, t}$ such that

$$
e^{g_{X, t}}=\frac{X_{t}}{X_{t-1}}
$$

are i.i.d. over time such that, for all $t$,

$$
g_{X, t}=g_{X} \text { with } g_{X} \sim N\left(\mu_{g_{X}}, \sigma_{g_{X}}^{2}\right)
$$

(A3) For all periods $t, K_{t}$ is independent of $g_{X, t}$ as well as i.i.d. over time. Furthermore, we make the-arguably strong-assumption that the covariance between the consumption-growth rate and the collusion structure is negligible, i.e., $\operatorname{cov}\left(g_{C, t}, K_{t}\right) \simeq 0$.

\section{Proposition 1.}

(i) There exists, for all $t \geq 1$, a period $t$ equilibrium price function $P_{t}: \Omega \rightarrow \mathbb{R}_{+}$if and only if

$$
-\delta+\mu_{g_{Z}}+\frac{1}{2} \sigma_{g_{Z}}^{2}<0
$$

whereby

$$
\begin{aligned}
\mu_{g_{Z}} & =2 \mu_{g_{X}}-\mu_{g_{C}} \\
\sigma_{g_{Z}}^{2} & =4 \sigma_{g_{X}}^{2}+\sigma_{g_{C}}^{2}-4 \sigma_{g_{X}, g_{C}} .
\end{aligned}
$$

(ii) If there exists a period $t$ equilibrium price function, then it is uniquely characterized by

$$
P_{t}^{*}(\omega)=\frac{e^{\left(-\delta+\mu_{g_{Z}}+\frac{1}{2} \sigma_{g_{Z}}^{2}\right)}}{1-e^{\left(-\delta+\mu_{g_{Z}}+\frac{1}{2} \sigma_{g_{Z}}^{2}\right)}} \cdot E\left[K_{t}^{-1}\right] \cdot\left(X_{t}(\omega)\right)^{2}
$$

for $\omega \in \Omega$. 
Observe that any volatility of the equilibrium prices (9) is exclusively driven by the volatility of the market-size which is the only random variable on the r.h.s. of equation (9). Now substitute the identity

$$
\left(X_{t}\right)^{2}=\frac{Y_{t}}{K_{t}}
$$

in (9) to obtain the following characterization of the equilibrium price-dividend ratio.

Corollary 1. The period $t$ price-dividend ratio is given by

$$
\frac{P_{t}^{*}}{Y_{t}}(\omega)=\frac{e^{\left(-\delta+\mu_{g_{Z}}+\frac{1}{2} \sigma_{g_{Z}}^{2}\right)}}{1-e^{\left(-\delta+\mu_{g_{Z}}+\frac{1}{2} \sigma_{g_{Z}}^{2}\right)}} \cdot E\left[K_{t}^{-1}\right] \cdot K_{t}
$$

for $\omega \in \Omega$.

Whenever the collusion structure remains constant over time the price-dividend ratio is given as the constant

$$
\frac{P^{*}}{Y^{*}}=\frac{e^{\left(-\delta+\mu_{g_{Z}}+\frac{1}{2} \sigma_{g_{Z}}^{2}\right)}}{1-e^{\left(-\delta+\mu_{g_{Z}}+\frac{1}{2} \sigma_{g_{Z}}^{2}\right)}}
$$

regardless of whether there is a lot or only little collusion in the industry. In contrast, if there is any volatility in the price-dividend ratio, then it must result from a volatile collusion structure whereby

$$
\operatorname{var}\left(\frac{P_{t}^{*}}{Y_{t}}\right)=\operatorname{var}\left(K_{t}\right)
$$

Furthermore, since $K_{t}$ tends to increase ${ }^{3}$ in the number of alliances $\# I_{t}$ in period $t$, we obtain, by (11), that a firm's price-dividend ratio tends to be high, resp. low, when there is less (resp. more) collusion in the industry. According to our model, a decrease (resp. increase) in price-dividend ratios indicates an increase (resp. decrease) in collusive behavior and vice versa. The economic intuition behind this finding is straightforward: In times of high resp. (low) collusion, profits (=dividend payments) are high (resp. low) whereas prices rather incorporate expected future profits that take subsequent changes in the collusion structure into account.

\section{Proof of Proposition 1}

We start by proving part (ii) first. Assume for the moment that the transversality condition is satisfied. Under the distributional assumptions A1-A3, we can then rearrange

\footnotetext{
${ }^{3}$ In particular, for the benchmark case of alliances of the same size we have that $K_{t}=\# i^{A}\left(I_{t}\right)$. $\left(\# I_{t}+1\right)^{2}$ is maximal if there is maximal competition (i.e., $\left.\# I_{t}=n, \# i^{A}\left(I_{t}\right)=1\right)$ and minimal if there is minimal competition (i.e., $\# I_{t}=1, \# i^{A}\left(I_{t}\right)=n$ ).
} 
the equilibrium price formula (7) as follows

$$
\begin{aligned}
P_{t}^{*} & =\sum_{s=t+1}^{\infty} E_{t}\left[M_{t, s} \cdot \frac{\left(X_{s}\right)^{2}}{K_{s}}\right] \\
& =\sum_{s=t+1}^{\infty} E_{t}\left[\beta^{s-t} \cdot\left(\frac{C_{s}}{C_{t}}\right)^{-1} \cdot\left(X_{s}\right)^{2}\right] \cdot E\left[K^{-1}\right] \\
& =\sum_{s=t+1}^{\infty} \beta^{s-t} \cdot E\left[e^{-g_{C} \cdot(s-t)} \cdot e^{2 g_{X} \cdot(s-t)}\right] \cdot\left(X_{t}\right)^{2} \cdot E\left[K^{-1}\right] \\
& =\sum_{s=t+1}^{\infty}\left(e^{-\delta}\right)^{s-t} E\left[e^{2 g_{X}-g_{c}}\right]^{s-t} \cdot E\left[K^{-1}\right] \cdot\left(X_{t}\right)^{2} .
\end{aligned}
$$

For notational simplicity let $g_{Z} \equiv 2 g_{X}-g_{C}$ and observe that

$$
g_{Z} \sim N\left(\mu_{g_{Z}}, \sigma_{g_{Z}}^{2}\right)
$$

with $\mu_{g_{Z}}$ and $\sigma_{g_{Z}}^{2}$ defined as in Proposition 1. That is, $e^{g_{Z}}$ is log-normally distributed, implying $E\left[e^{g_{Z}}\right]=e^{\left(\mu_{g_{Z}}+\frac{1}{2} \sigma_{g_{Z}}^{2}\right)}$. Substitution in (16) gives

$$
P_{t}^{*}=\sum_{s=t+1}^{s-t} e^{\left(-\delta+\mu_{g_{Z}}+\frac{1}{2} \sigma_{g_{Z}}^{2}\right) \cdot(s-t)} \cdot E\left[K^{-1}\right] \cdot\left(X_{t}\right)^{2}
$$

Finally, an application of the present value formula gives (9) whenever (8) holds.

Ad (i). By the proof of part (ii), if there exists a period $t$ equilibrium price function, then it is given by (7). In order to prove existence, it remains to be shown that the transversality condition is indeed satisfied, i.e., $\lim _{s \rightarrow \infty} E_{t}\left[M_{t, s} \cdot P_{s}^{*}\right]=0$ such that, for all $s \geq 1, P_{s}^{*}$ is given by $(7)$. Observe that

$$
\begin{aligned}
E_{t}\left[M_{t, s} \cdot P_{s}^{*}\right] & =E_{t}\left[\beta^{s-t} \cdot e^{-g_{C} \cdot(s-t)} \cdot \frac{e^{\left(-\delta+\mu_{g_{Z}}+\frac{1}{2} \sigma_{g_{Z}}^{2}\right)}}{1-e^{\left(-\delta+\mu_{g_{Z}}+\frac{1}{2} \sigma_{g_{Z}}^{2}\right)}} \cdot E\left[K^{-1}\right] \cdot\left(X_{s}\right)^{2}\right] \\
& =\frac{e^{\left(-\delta+\mu_{g_{Z}}+\frac{1}{2} \sigma_{g_{Z}}^{2}\right)}}{1-e^{\left(-\delta+\mu_{g_{Z}}+\frac{1}{2} \sigma_{g_{Z}}^{2}\right)}} \cdot\left(e^{-\delta)^{s-t}} E\left[e^{2 g_{X}-g_{c}}\right]^{s-t} \cdot E\left[K^{-1}\right] \cdot\left(X_{t}\right)^{2}\right. \\
& =\frac{e^{\left(-\delta+\mu_{g_{Z}}+\frac{1}{2} \sigma_{g_{Z}}^{2}\right)}}{1-e^{\left(-\delta+\mu_{g_{Z}}+\frac{1}{2} \sigma_{g_{Z}}^{2}\right)}} \cdot e^{\left(-\delta+\mu_{g_{Z}}+\frac{1}{2} \sigma_{g_{Z}}^{2}\right) \cdot(s-t)} \cdot E\left[K^{-1}\right] \cdot\left(X_{t}\right)^{2}
\end{aligned}
$$

so that

$$
\lim _{s \rightarrow \infty} E_{t}\left[M_{t, s} \cdot P_{s}^{*}\right]=\frac{e^{\left(-\delta+\mu_{g_{Z}}+\frac{1}{2} \sigma_{g_{Z}}^{2}\right)}}{1-e^{\left(-\delta+\mu_{g_{Z}}+\frac{1}{2} \sigma_{g_{Z}}^{2}\right)}} \cdot E\left[K^{-1}\right] \cdot\left(X_{t}\right)^{2} \cdot \lim _{s \rightarrow \infty} e^{\left(-\delta+\mu_{g_{Z}}+\frac{1}{2} \sigma_{g_{Z}}^{2}\right) \cdot(s-t)} .
$$

That is, the transversality condition is satisfied if and only if (8) holds. 


\section{References}

Bester, H. and E. Petrakis (1993) "The Incentives for Cost Reduction in a Differentiated Industry," International Journal of Industrial Organization 11, 519-534.

Bolotova, Y., Connor, J., and D. Miller (2008) "The Impact of Collusion on Price Behavior: Empirical Results from Two Recent Cases," International Journal of Industrial Organization 26, 1290-1307.

Bonanno, G. and B. Haworth (1998) "Intensity of Competition and the Choice Between Product and Process Innovation," International Journal of Industrial Organization 16, 495-510.

Lucas, R.E. Jr. (1978) "Asset Prices in an Exchange Economy," Econometrica 46, 1429-1445.

Mehra, R., and E.C. Prescott (1985) "The Equity Premium: A Puzzle," Journal of Monetary Economics 15, 145-161.

Tirole, J. (1988) The Theory of Industrial Organization, MIT Press: Cambridge. 